\title{
Fluidic Control of Molten Metal Flow
}

V. Tesař

Fluidic devices built from suitable refractory materials can withstand the extreme conditions encountered in flows of molten metals - even those with a high melting point. This paper informs about the hydrodynamic aspects of a recent development of a fluidic controller keeping the metal gravity flow constant. The controller uses a narrow notch weir for generating the control signal and a fluidic vortex valve as the actuator. An unusual feature is the signal transfer between the two components performed also by a molten metal flow. It is even possible to amplify the signal using the liquid metal as the working medium in the amplifier.

Keywords: metallurgy, fluidics, vortex amplifier, jet-deflection amplifier.

\section{Introduction}

Metals are made and processed in furnaces, which usually operate on a batch basis. For continuous technological processes involving molten metals, the material is temporarily stored in some vessel - either the furnace itself or in some periodically re-filled tundish - which supplies the processing plant by gravity flow through an orifice located near its bottom. Of course, the gravity flow rate varies with the changing level of the metal inside the vessel as it is drained or re-filled. In some processes this variation causes considerable problems. Only a very crude form of flow control is currently used as a remedy. A simple valve is formed by a conical body of refractory material immersed in the melt at the upstream side of the orifice and held there by an external mechanical linkage. Manually moving the body into and out from the orifice changes the flow rate by varying the available flow cross section. The achievable constancy depends critically upon the dexterity and experience of the worker who operates this valve - usually in quite adverse environment. Attempts at applying automatic control have never been successful, the extreme temperature making the operating conditions too difficult. Also sensing the actual flow rate (or metal height inside the furnace) is a difficult problem.

In the present project, flow control is achieved using purely fluidic elements. These are flow handling devices that (in contrast to traditional mechano/fluidic devices, acting on the fluid by mechanical, moving components) possess no moving parts. They act upon the controlled flow solely by hydrodynamic phenomena generated in constant-geometry cavities. Fluidic devices have been demonstrated earlier (as described, e.g., in ref. [2] and [3]) to work successfully at very high temperatures. They are robust and reliable. A fluidic controller built of them is compact, and the shape of its parts is simple enough to enable them to be built them from refractory materials.

\section{The task and the solution}

The present fluidic controller was developed for Atomising Systems Limited, Sheffield S9 1EW, England - a manufacturer of fine metal powders as well as of the machinery used in metal powder production. The machinery is built to process, depending upon size, from $400 \mathrm{~kg}$ up to $1000 \mathrm{~kg}$ of liquid metal per minute. This is the flow that has to be handled by the controller, which should be able to keep the flow rate constant to within $5 \%$ while the molten metal level height in the tundish varies between $H=1$ m maximum and $H=0.25 \mathrm{~m}$ minimum. To give an idea about the properties of the processed molten metals, a $1200-1400^{\circ} \mathrm{C}$ melt may have a typical density around $7 \cdot 10^{3} \mathrm{~kg} / \mathrm{m}^{3}$ and kinematic viscosity near to $1 \cdot 10^{-6} \mathrm{~m}^{2} / \mathrm{s}$.

The fluidic controller consists of two essential parts (Fig. 1): an actuating valve in the main flow branch, and a sensor connected to the valve by a signal transfer pipe. The extraordinary feature here is the signal being carried by the

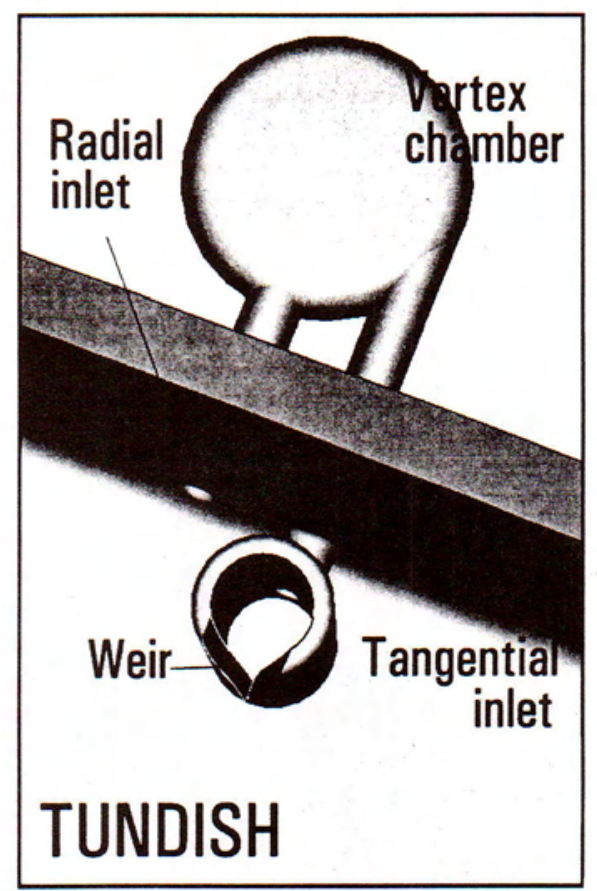

Fig. 1: The initial layout of the fluidic controller for maintaining a constant molten metal flow from a tundish. View from above.

molten metal flow - certainly a highly unusual signal carrying medium, but fully in line with the usual tendency of fluidics to employ the working fluid for signal transfer and processing. The valve is a fluidic vortex amplifier [1]. It turns down the flow by centrifugal acceleration effects acting on the molten metal, induced to rotate in an axisymmetrically shaped vortex chamber by the control signal flow, which enters the vortex chamber tangentially (Fig. 2). 


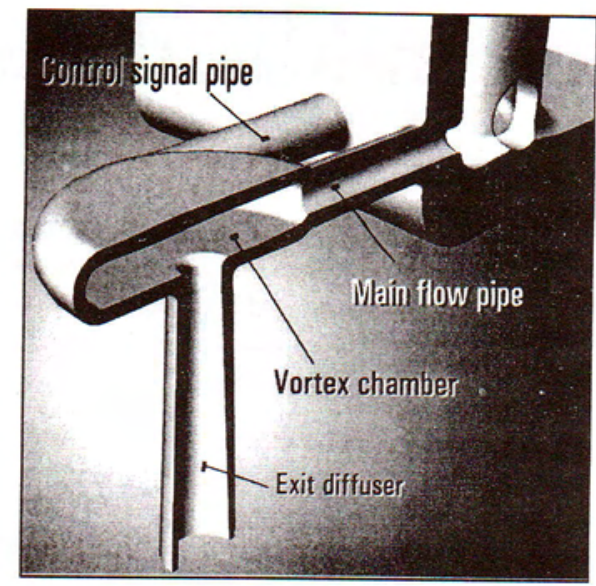

Fig. 2: Initially, the vortex valve had this flat vortex chamber. Rotation of the molten metal in the chamber is caused by the control signal flow, entering the chamber tangentially.

\section{Sensing}

The sensor, responding to the variations of the surface level in the tundish, is formed by the narrow cut-out in its side wall of the tangential branch inlet (Fig. 1, Fig. 3). The cut-out acts as a weir of variable cross section, dependent upon the molten metal surface height. The flow rate that it passes through decreases more sharply with decreasing molten metal level than the flow in the main inlet, which enters the vortex chamber radially. The actual weir design is somewhat complicated by the concentric well with entrance

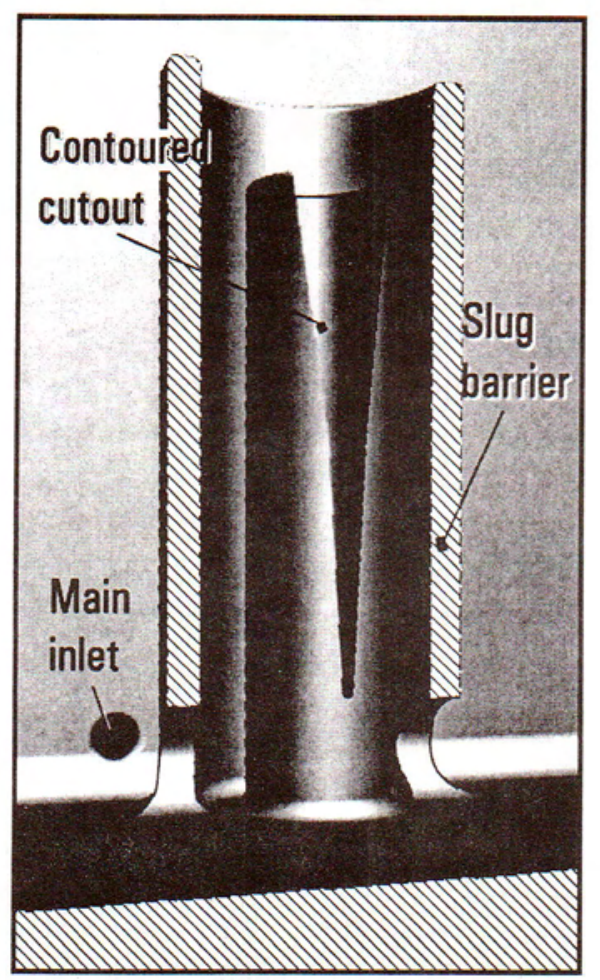

Fig. 3: The sensing of the molten metal level by the narrow weir inside the tundish. This is a version with the slag barrier well, half of which is here cut away to show the internal arrangement. holes at the bottom, Fig. 3. This protects the weir notch from ingress of slag, often floating on the molten metal surface.

It is a remarkable fact that a weir overflow can easily be set up (by increasing the cross section in the vertical direction) to reduce the pressure head variations - up to keeping it essentially constant, irrespective of the flow rate. It cannot perform the opposite task of reducing the variations in the flow rate - it would be useless to try with the cross section decreasing in the vertical direction. Of course, in the present case the desirable action is exactly this very opposite task of keeping the flow constant irrespective of the varying head. No weir can achieve this. Fortunately, the vortex amplifier possesses in principle the signal inverting capability. Its output flow decreases with increasing tangential control flow - and this is what is employed in the present controller. The weir is used as the inversely operating (pressure head maintaining) variable restrictor (Fig. 4) in the control signal transfer branch.

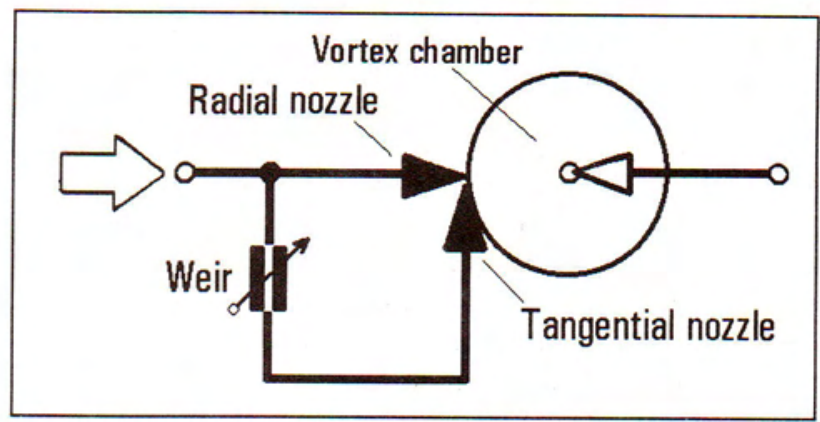

Fig. 4: Schematic circuit diagram of the simplest flow controller version. The weir represents a level-height dependent variable restrictor in the tangential flow branch.

The vortex valve starts operating, when the tundish is initially full, in the "CLOSED" regime - with full tangential control flow, Fig. 5. As the level in the drained tundish goes down, the tangential inlet becomes more and more restricted. The intensity of the rotation inside the vortex chamber gradually decreases. Finally, the turning-down effect ceases com-

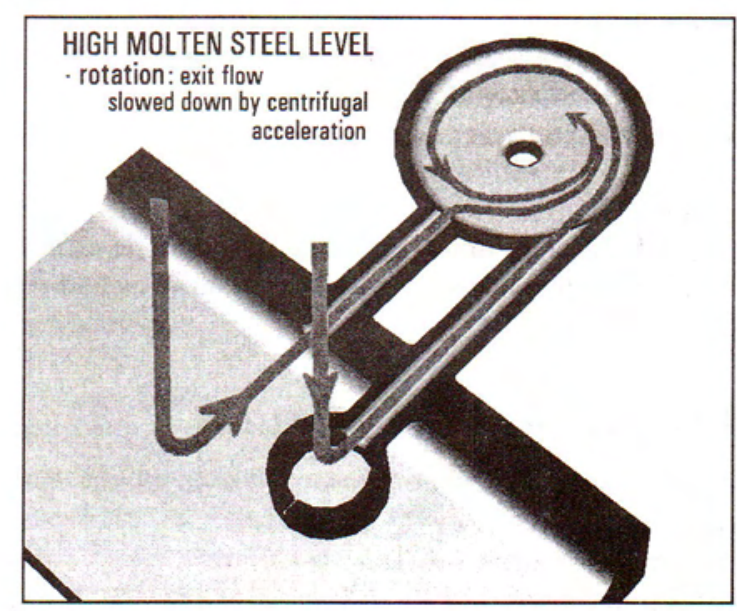

Fig. 5: Horizontal section through the vortex chamber and its inlets, showing both radial and tangential flow at a high level of the metal in the tundish 


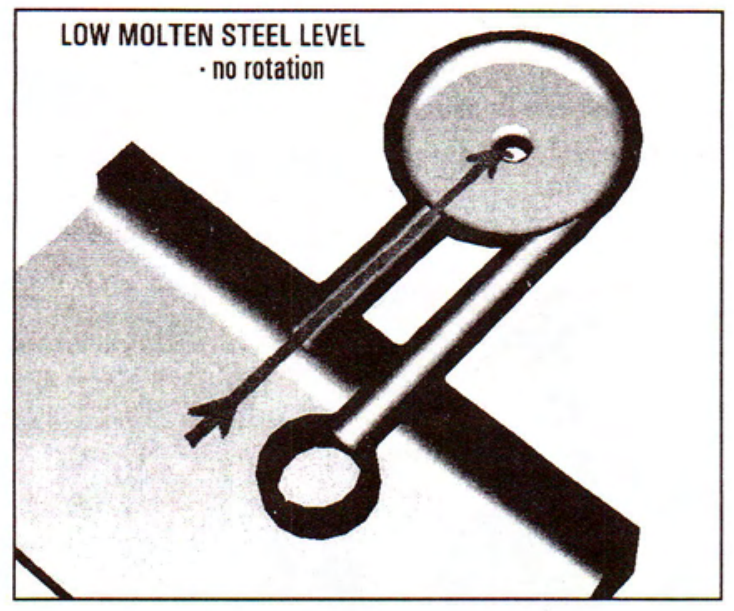

Fig. 6: Horizontal section with schematic representation of radial flow only, as it takes place at a low level inside the tundish

pletely and the valve enters the "OPEN" regime (Fig. 6), with only radial flow admitted. As shown in Fig. 7, this leads to the required nearly vertical characteristic - but not everywhere, only during the transition between the "CLOSED" and the "OPEN" regimes. Obviously, this turning-down control principle cannot maintain the constant flow rate down to the very last drop of metal in the tundish. This, fortunately, is not required in the present application: the tundish is re-filled by the new charge of molten metal before the controller operation reaches the "OPEN" regime.

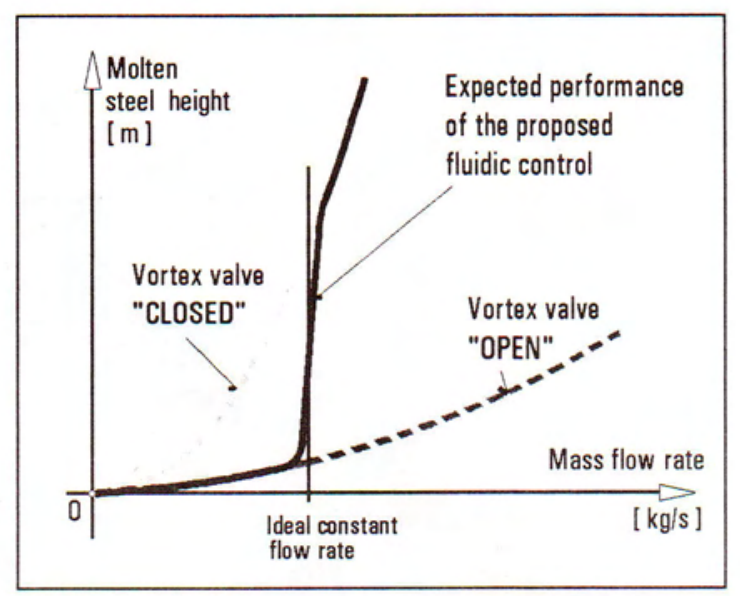

Fig. 7: Characteristic: the dependence between flow rate and level height in the tundish. The control action takes place during the transition from the "CLOSED" regime (both inlets active) to the "OPEN" regime (radial inlet flow only).

\section{Initial valve design}

The valve is the critical component. The weir design is a relatively straightforward task as long as the valve can be brought into the proper "CLOSED" and "OPEN" regimes at the ends of the vertical transition segment of the characteristic.

For the initial tests the controller was placed externally, outside the tundish, while the weir was inside, Fig. 1. This location of the valve is not practical from the operational point of view - the delicate device may easily be damaged and it may be necessary to arrange for it to be heated to prevent metal solidification inside its cavities. This was neglected in the initial phase of the research, the primary consideration being easy access to the valve during the tests. Also the size was smaller than for the final version: it was aimed at processing only $600 \mathrm{~kg} / \mathrm{min}$ of metal. The actual mean mass flow rate in the test was $10.12 \mathrm{~kg} / \mathrm{s}$ for both inlets fully open at the metal level height in the tundish at its $H=1 \mathrm{~m}$ maximum. The pipe diameters were $26 \mathrm{~mm}$ (radial) and $29 \mathrm{~mm}$ (tangential). The vortex chamber was flat, of $240 \mathrm{~mm}$ internal diameter - slightly larger than 9 inlet pipe diameters - with $18 \mathrm{~mm}$ radius rounded periphery. The central exit orifice diameter was $17 \mathrm{~mm}$.

The first objective of establishing the proper operation in the "CLOSED" regime was met surprisingly well. The turning down by "centrifugal" acceleration was found to be remarkably efficient. Perhaps even more surprisingly, the valve failed completely in what was expected to be the much less problematic "OPEN" regime. Its resistance to the flow was excessively high. The explanation was found by CFD solutions of the flowfield in the valve.

\section{The "bathtub vortex" problem}

The convergence history of the computations in the problematic "OPEN" state exhibited oscillations indicative of loss of stability. After a disproportionally long solution time, the computation converged to a solution in which the initial bifurcation of the flow into two contra-rotating vortices gave way to an asymmetry. One of the vortices became larger, tending to occupy the centre of the chamber. The instability then made it to grow until it filled the chamber completely, while the other vortex disappeared. In the finally converged solution there was a single strong central vortex effectively blocking the flow, seen in the computed pathlines for the converged state in Fig. 8.

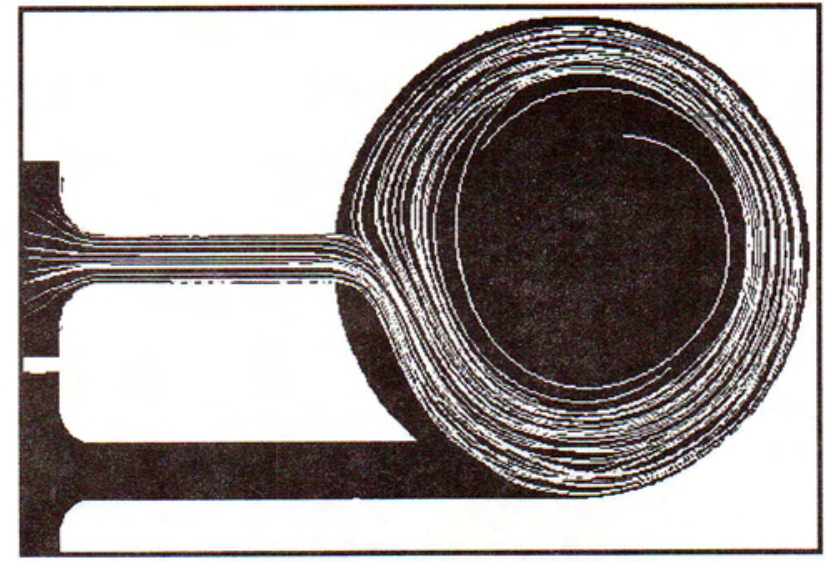

Fig. 8: The "bathtub vortex" instability: even with purely radial flow, vortical motion was spontaneously generated in the original flat vortex chamber, deteriorating the "OPEN" regime performance

This instability effect, in fact, should not be surprising. It is a phenomenon well known from draining bathtubs or wash basins. In actual operation, the inability to obtain a symmetric 
"OPEN" state is more dangerous than in the computation starting from a symmetric initial flow. The usual operating mode is the valve starting from the high-rotation "CLOSED" state with the full tundish, gradually opening as the liquid metal level decreases. The "bathtub effect" would cause the rotation to remain even without any tangential flow.

A similar highly undesirable effect was encountered by the author in his development of vortex amplifiers for UJV Řež (Institute of Nuclear Research, Czech Republic) in the 1980 's. The remedy applied at that time was the vortex chamber arrangement shown in Fig. 9. The radial flow comes into the chamber, instead of through the single radial inlet, by several (four in Fig. 9) radial inlets heading towards the central exit hole symmetrically from several locations on the vortex chamber perimeter. Unfortunately, a similar layout with the flow splitting into a number of paths would make the geometry of the molten metal flow controller too complex for construction with refractory materials.

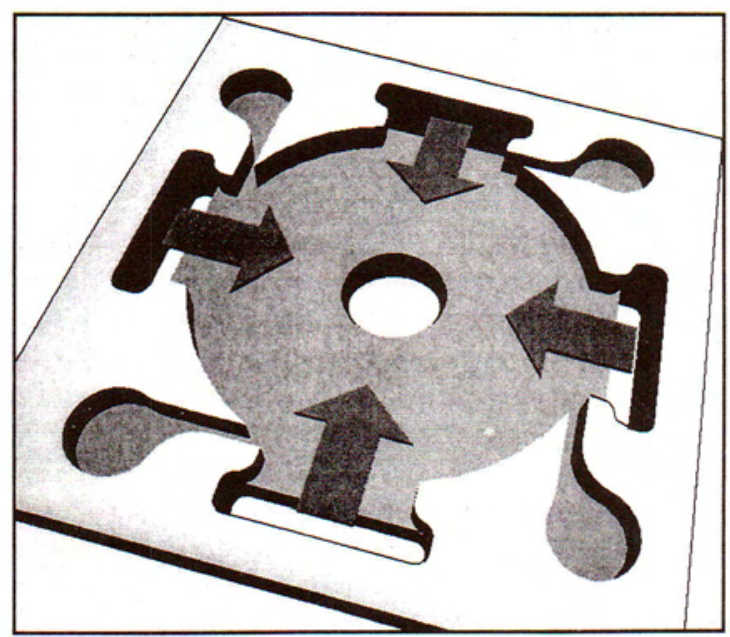

Fig. 9: Standard way to suppress "bathtub vortex" instability: symmetric radial inflow from many (here four) directions

\section{The conical chamber}

Another approach, which was finally selected to eliminate the "bathtub vortex", was inspired by the conical chambered vortex devices developed by Prof. Brombach [4]. The idea is to shape the vortex chamber so as to form the hollow cone shown in Fig. 10. The radial fluid flow is "funnelled" into the

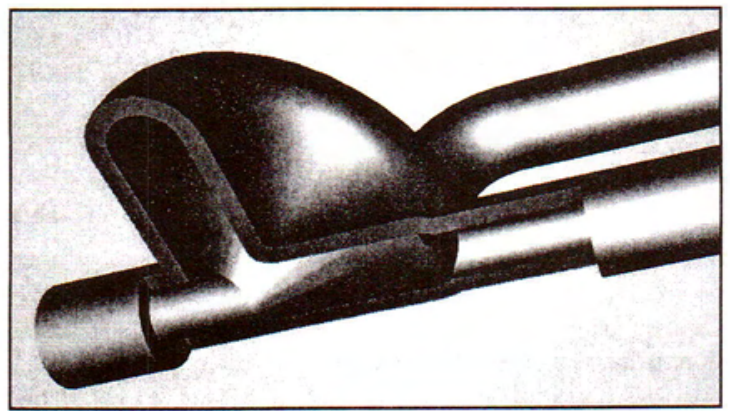

Fig. 10: A conical vortex chamber was found effective in suppressing the "bathtub vortex" without complex multi-pipe radial inlets exit, which is inclined to make the entry easier. An additional advantage of this configuration is that the whole flow control system is more compact.

Several versions of the conical vortex valves, e.g., the particularly compact version with the small $45^{\circ}$ cone vertex half-angle shown in Fig. 11, were investigated, mainly by CFD

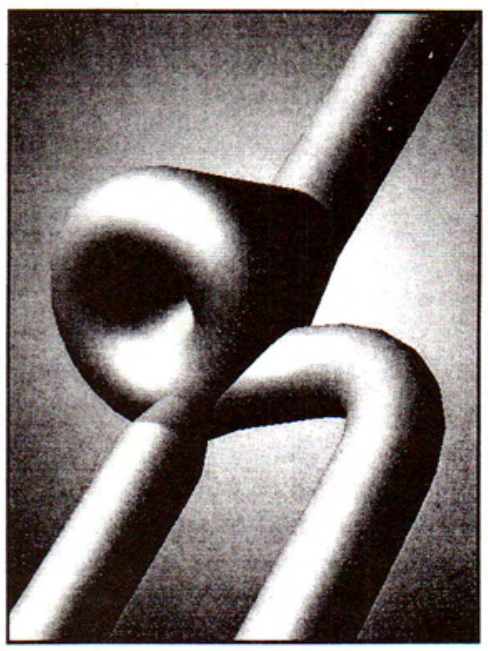

Fig. 11: A version of the tested conical vortex valve, with nozzle contraction in the radial inlet. The obvious advantage of a conical valve is its compactness and small dimensions. This is the shape of the "lost wax" core (in fact an ACIS model) - a "negative" of the valve cavities.

solutions. Fig. 12 presents an example of the used discretisation grid. Typically, the grid possessed about 120000 unstructured tetrahedral elements (the number changed by adaptive refinement of the grid in the course of the solutions).

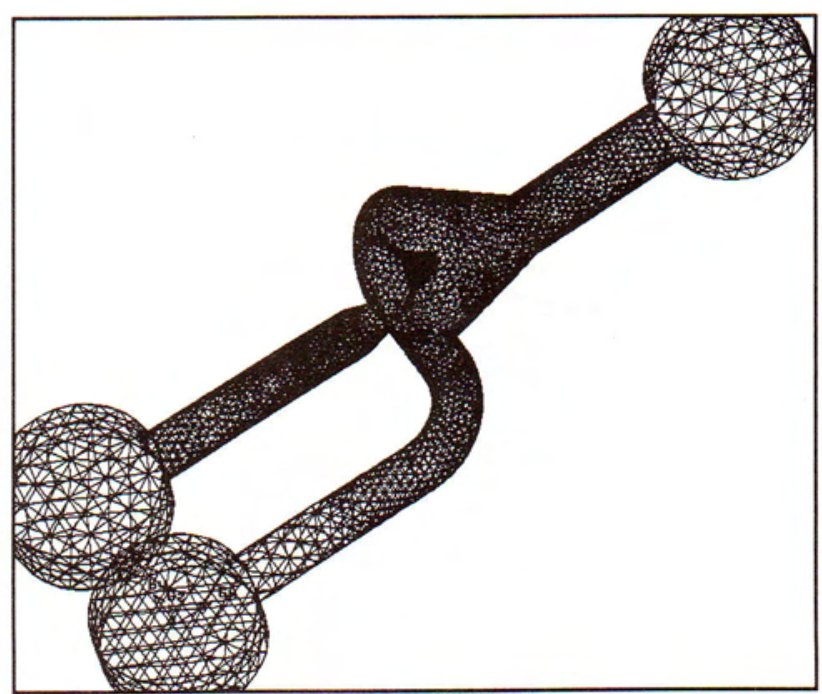

Fig. 12: The discretisation grid used in numerical computations of the conical vortex valve flowfield. The end chambers at the inlets are added for better definition of the boundary conditions there.

The solutions, using FLUENT 5, employed a two-equation "rngke" turbulence model with standard values of the model constants and handling the low Re turbulence by the renormalisation group approach. Earlier favourable experience 


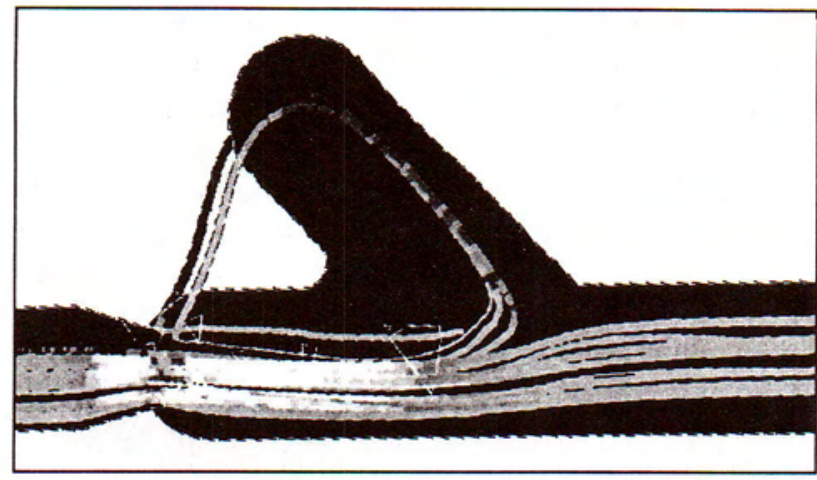

Fig. 13: Pathlines computed in the "OPEN" state regime for the conical valve. The two vortices leading to the instability here degenerate into harmless weak sideways loops.

with the "CLOSED" regime has led to concentrating overly on the "OPEN" regime flow, for which finally quite satisfactory results were obtained (Fig. 13). Unfortunately, as shown in Fig. 14, the layout with the small vortex chamber diame-

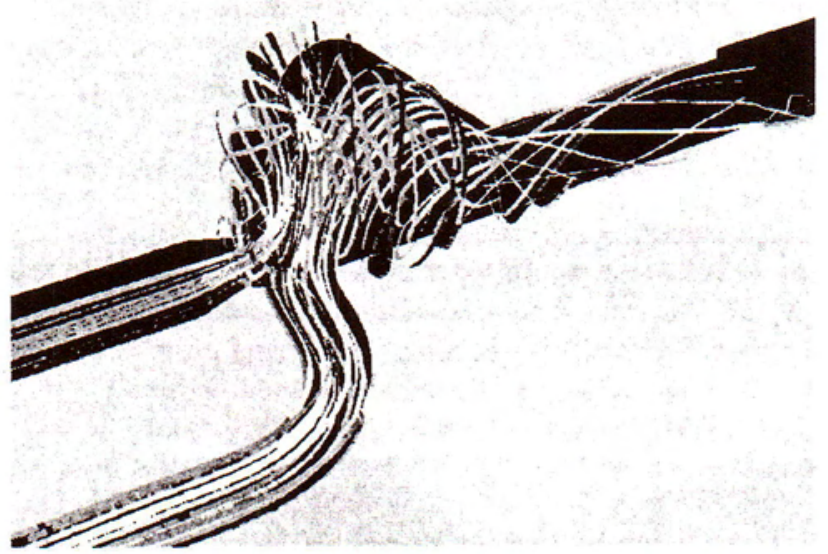

Fig. 14: Computed pathlines in the conical-chamber vortex valve with the same input pressure applied to both radial and tangential inlets. The turning down by rotation was not very effective, as seen from the absence of co-ordinated rotation.

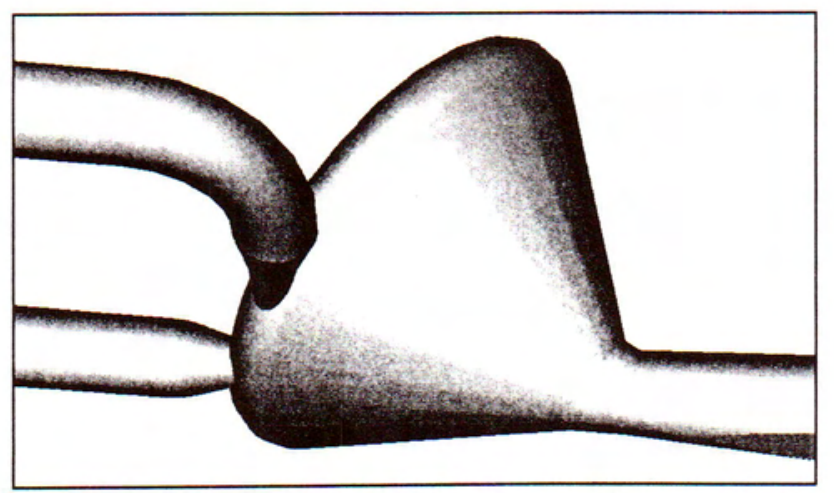

Fig. 15: The latest vortex chamber configuration to handle the $16.7 \mathrm{~kg} / \mathrm{s}(=1000 \mathrm{~kg} / \mathrm{min})$ goal. Pipe diameters $60 \mathrm{~mm}$, the fluid entering tangentially is also accelerated in a nozzle contraction. The radius of the chamber was increased to $380 \mathrm{~mm}$ for improvement in the rotational state. ter then exhibited a deterioration in the "CLOSED" regime performance. The art of designing fluidic devices is to find the proper balance between the opposing requirements of the different operating conditions. The latest, most successful compromise design to handle the largest required $1000 \mathrm{~kg} / \mathrm{min}$ flow rate is shown in Fig. 15. The diameter of the vortex chamber was increased to nominal $380 \mathrm{~mm}$ - with the inlet pipe diameters nominally $60 \mathrm{~mm}$. The larger diameter, with the longer distance between the radial inlet and the central exit - together with larger, $80^{\circ}$ cone vertex half-angle - required for the radial flow to be accelerated in the nozzle. This, in turn, called for another nozzle in the tangential inlet, to improve the momentum imparted in the "CLOSED" state.

\section{Version with jet-deflection amplifier}

The improvement achievable by amplification in the signal transfer of paths is an elementary fact of control systems theory - the classical derivation is, e.g., in [5]. In the present case the signal amplification can improve the steepness of the desirably nearly vertical transition path between the "CLOSED" and "OPEN" states shown in Fig. 7. Recent progress in modern refractory materials and the relative simplicity of fluidic jet-type amplifiers made possible an investigation of the improvements achievable by inserting a proportional amplifier [6] into the inlet pipes, as shown schematically in Fig. 16. Indeed, the arrangement, as seen in Fig. 17 (view-

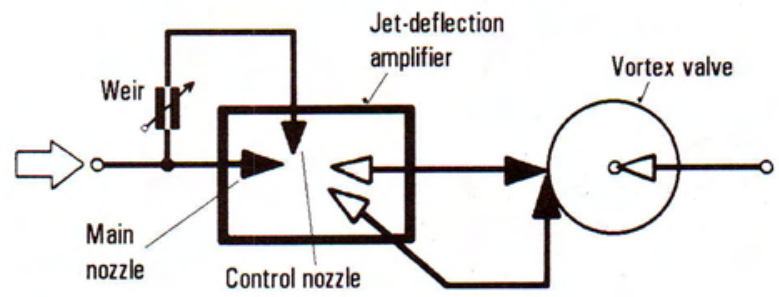

Fig. 16: Schematic representation of the more complex version of the fluidic control with the jet-deflection amplifier.

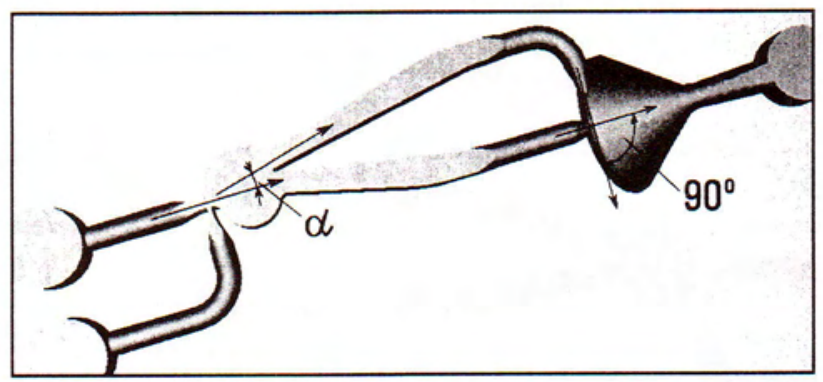

Fig. 17: Jet deflection amplification. Interaction of the two incoming flows may lead to a small resultant deflection angle $\alpha$-but the amplifier translates it into a change by a full $90^{\circ}$ at the entrance into the vortex chamber.

ed from below) does not represent a substantial increase in overall complexity - despite the unpleasant fact that the fluidic jet-deflection amplifiers require rectangular cross sections of the cavities which did not blend easily with the original circular cross-sections of the pipes, taken over from the earlier versions. 
Perhaps the simplest explanation of the benefits obtained by the amplifier is presented in Fig. 17 in terms of the angle of the deflection of the resultant flow generated by the perpendicular impact of the two inlet flows. In the vortex valve we need the greatest possible tangential resultant inflow to get it into the fully "CLOSED" state - but the interaction of the two flows leads to a relatively small angle $\alpha$. In fact, standard vortex amplifiers have to be controlled by a much higher control pressure applied in the tangential inlet, which is not available in the present case. The amplifier accepts this small deflection angle and sends the resultant flow into the vortex chamber at the full $90^{\circ}$ deflection angle. Of course, this large inclination of the flow from the amplifier also helps in all intermediate states between this "CLOSED" state and the "OPEN" state.

Unfortunately, there is very little available information about the proportional unvented jet deflection amplifiers needed for the present purpose. Much more is known about the switched bistable or monostable [2] versions, utilising the attachment of the jet to one of the walls of the interaction chamber [6]. This, however, is exactly what had to be avoided here (it would mean a hysteresis in the characteristics). The design of the amplifier was therefore guided by the idea of an interaction chamber with walls bulged out, away from the jet paths. This has led to a nearly circular cavity, as seen in the pictures immediately downstream from the location where the exits of the main nozzle and the control nozzle meet. So as not to increase the overall pressure drop in the "OPEN" state by the addition of the amplifier, its size was chosen rather large [7]. While the round pipes are of diameter $d=58.96 \mathrm{~mm}$, i.e., of cross sectional area $2730 \mathrm{~mm}^{2}$, the main nozzle exit width is $b=26.26 \mathrm{~mm}$. The nozzle height is $h=58.96 \mathrm{~mm}$, (equal to the diameter of the pipes) so that the cross-sectional area of the nozzle exit is $1548 \mathrm{~mm}^{2}$. This represents 0.567 area contraction in the main nozzle. The distance of the splitter from the nozzles is $203.3 \mathrm{~mm}$, which represents 7.742 nozzle exit widths. The radial path collector for capturing the jet in the "OPEN" regime was placed directly opposite the main nozzle, separated by a simple splitter with a slightly rounded tip from the tangential branch collector. Further downstream from the collectors are long diffusers - their length is dictated by the necessity of small diffuser divergence angles.

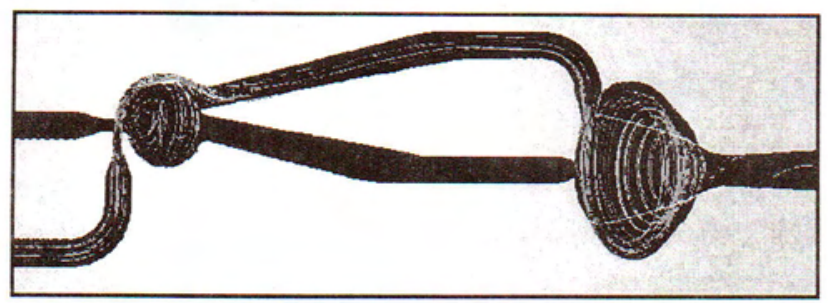

Fig. 18: Computed pathlines corresponding to the diagnostic test performed to validate the amplifier behaviour. Only the control inlet is active: it is satisfactory to see that this leads to purely tangential flow into the vortex valve and a full rotation in its chamber.

It is quite common in fluidics that the initial designs, guided more or less by the designer's intuition, do no perform perfectly and require further development. This is also the present case. In particular, experience has shown that the interaction cavity was too small, generating pressure fields and feedback loop flows deflecting the resultant jet away from the desirable directions.

No problem was encountered in the case shown in Fig. 18, with only control flow applied (corresponding to $N$ in Fig. 20). This sent all the flow (shown in Fig. 18 by the computed pathlines) into the tangential inlet of the vortex valve. What is needed in the present application, however, is to get also this

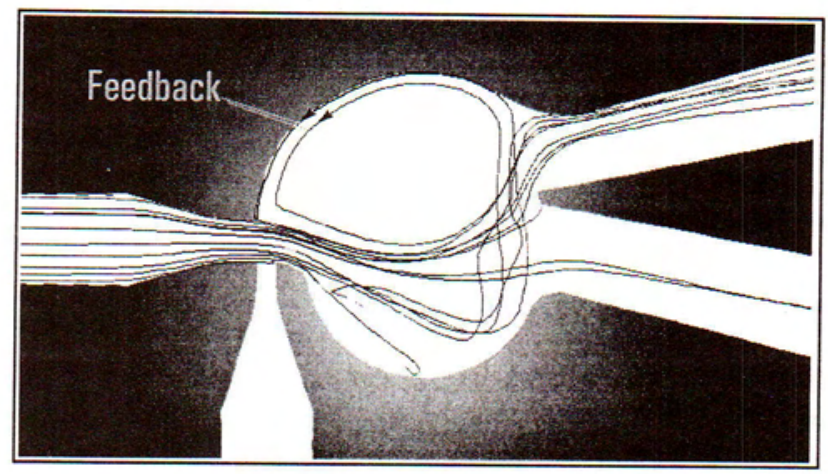

Fig. 19: Detail of the computed streamlines in the interaction cavity of the amplifier without any control flow. Instead of the expected straight flow into the preferred collector the unwanted internal feedback loop causes an undesirable flow into the tangential branch.

tangential flow if there is a simultaneous flow into the main nozzle - generated by the same $1 \mathrm{~m}$ metal level height. With the initial amplifier design, a part of the flow managed to get into the main collector, resulting in reduced intensity of rotation in the vortex chamber. Problems were also encountered in the "OPEN" regime, when all the flow from the main nozzle is required to reach the main, radial branch collector. As shown in Fig. 19, even the apparently simplest goal of a straight jet flow was initially not achieved. The resultant flow became bent due to the pressure difference across the jet and due to the action of the feedback loop flow. The consequence

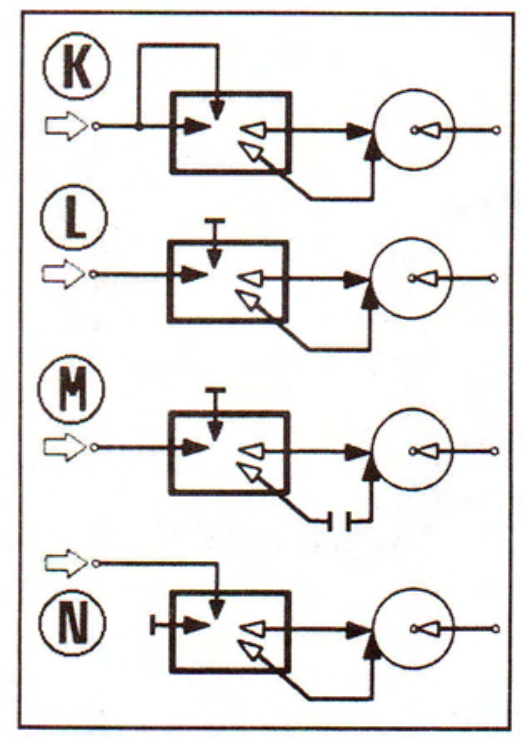

Fig. 20: Schematic representation of the four test conditions in Fig. 21 


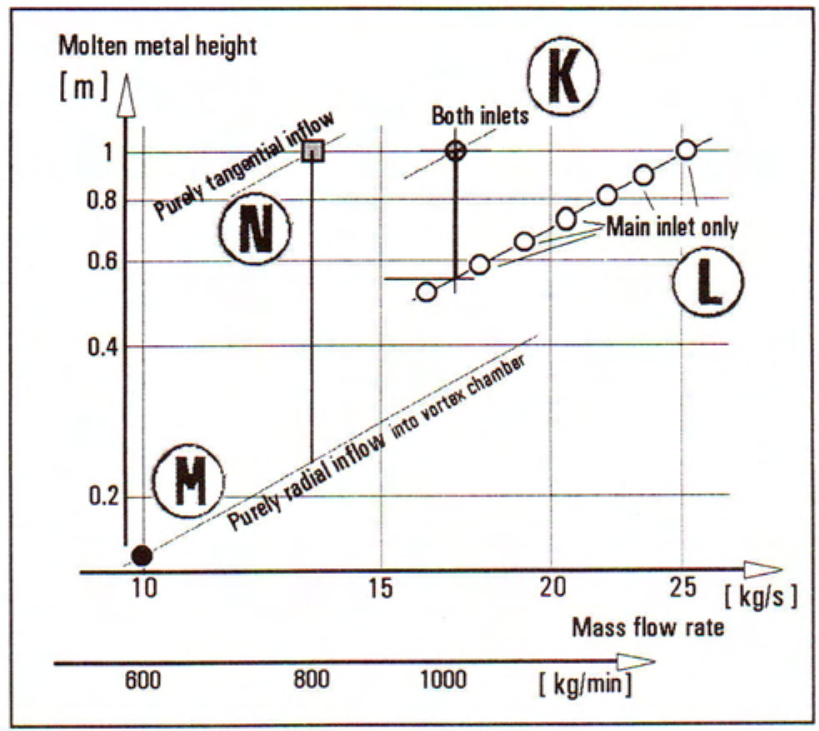

Fig. 21: Results of several tests with the control involving the amplifier (Fig. 17), plotted in logarithmic co-ordinates as the dependence between flow rate and metal level height

was the unwanted rotation in the vortex chamber. These problems reduced the achieved performance, as shown in Fig. 21 presenting the resultant total mass flow rates and heights of the molten metal inside the tundish, plotted in logarithmic co-ordinates. Nevertheless, even in this first, far from perfect design, the controller could operate in the intended role, though in a smaller range of acceptable heights. It could keep a perfectly constant flow $1000 \mathrm{~kg} / \mathrm{min}$ between the level heights $H=1 \mathrm{~m}$ (i.e. from point $K$ in Fig. 21) and $H=0.55 \mathrm{~m}$ (an interpolation between the points $L$ ).

An improvement of the flow in the amplifier to get the proper "OPEN" state, as seen in Fig. 21 by comparison with the curve passing through the artificially obtained point $M$,

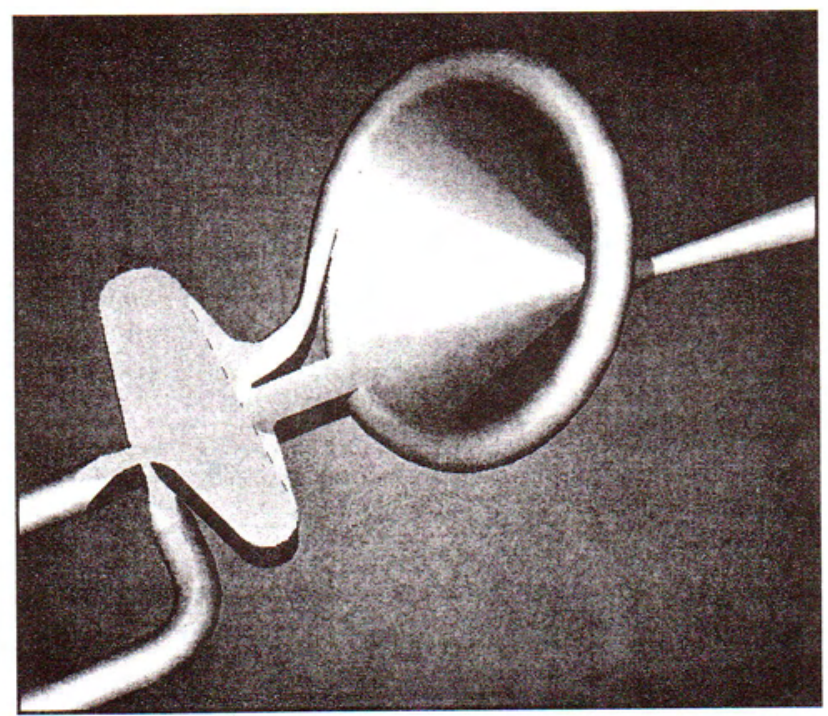

Fig. 22: The final version of the $1 \mathrm{t} / \mathrm{min}$ controller (ACIS mode "negative" of the cavities). The amplifier integrated into the vortex chamber entrance has its interaction cavity re-shaped and increased to suppress the generation of feedback loop flows. could reduce the "OPEN" operating point to $H=0.33 \mathrm{~m}$. This was obtained by adjusting the shape of the amplifier cavity. The improvements finally led to the layout shown in Fig. 22. Using the same vortex chamber shape as before, the desired performance values were achieved: the flow rate $1000 \mathrm{~kg} / \mathrm{min}$ is kept constant between the heights $H=1 \mathrm{~m}$ and $H=0.25 \mathrm{~m}$.

\section{Conclusions}

Modern graphite-based refractory materials make it possible to build fluidic devices with a reasonably long life even for handling the flow of high melting point metals, such as steel. Indeed, the most unusual feature of the discussed controllers - signal transfer and processing (sign inversion and amplification) in molten metal flows - is realisable only thanks to the progress in the materials and manufacturing side of the project. The hydrodynamics discussed here does not actually bring something very special - and the concept may be equally well used to control flow rates of other fluids that are difficult to handle, such as molten salts or the radioactive and corrosive fluids handled in nuclear fuel re-processing.

Turning down by the "centrifugal" acceleration in rotating fluids was found effective enough, and the problems that were encountered - perhaps somewhat surprisingly - were those limiting the performance in the "OPEN", non-rotational regimes. In particular, there was a problem of "bathtub vortex" instability. The present unusual conical shape of the vortex chamber seems to provide a successful solution.

\section{Acknowledment}

The author is thankful to John J. Dunkley, technical director of Atomising Systems Limited, Sheffield, for suggesting the problem and for his continuing encouragement.

\section{References}

[1] Tesař, V.: Großmaßstäbliche fluidische Ventile für die Durchflußsteuerung. Messen-steuern-regeln Bd. 26, Nr. 4, Berlin 1983.

[2] Tesař, V.: Fluidic Load-Switched Valve for Hot Gas Flow Control. Proceedings of FLUCOME'94 - the $4^{\text {th }}$ Triennial International Symposium on Fluid Control, Fluid Measurement and Visualization, Toulouse (France), September 1994, p. 741.

[3] Tesař, V., König, A., Macek, J., Baumruk, P.: New Ways of Fluid Flow Control in Automobiles: Experience with Exhaust Gas Aftertreatment Control. Proc. of World Automobile Congress, Paper No. F2000H192, Seoul (Korea): Society of Automotive Engineers, ISBN 89-85000-00-4 98550, June 2000, p. 167.

[4] Festschrift UFT 2002. Publ. By UFT Umwelt- und Fluid-Technik, Bad Mergentheim (Germany), June 2002.

[5] Tsien, H. S.: Engineering Cybernetics. New York: McGraw-Hill Book Company, 1954.

[6] Tesař, V.: Valvole fluidiche senza parti mobili. Oleodinamica - pneumatica, revista delle applicazioni fluidodinamiche, Milano 1998, No. 3 - marzo, Anno 39, p. 216.

[7] Tesař, V.: Optimum Loading of Fluidic Unvented Jet-Deflection Valves. Proceeding of Internat. Conf. Riadenie teku- 
tinových systémov 97', Slovakia: Publ. by University of Žilina, ISBN 80-7100-452-9, November 1997, p. 25-29.

Prof. Ing. Václav Tesař, CSc.

phone: +441142227551

fax: +441142227501

e-mail:v.tesar@sheffield.ac.uk

Process Fluidics Group

Department of Chemical and Process Engineering

The University of Sheffield

Mappin Street

Sheffield S1 3JD, United Kingdom

(on leave from Czech Technical University in Prague) 